Conclusion Appropriate training, a robust competency framework and ongoing support is key to safe, effective and appropriate medication administration by healthcare assistants allowing patients to die in their preferred place of care.

\section{P-126 REDUCING THE RISK OF FALLS AND PROMOTING INDEPENDENCE}

Elspeth McGloughlin. Kirkwood Hospice, Huddersfield, West Yorkshire

10.1136/bmjspcare-2018-hospiceabs.151

Falls are common across older populations and in particular for people who have life- limiting illnesses. Our organisation wanted to reduce the risk of falls and minimise harm to patients whilst promoting independence, dignity and enablement across our in-patient unit and day therapy services. We felt the most effective way to implement multifactorial assessment and intervention recommended by NICE would be to adopt and utilise a hospice wide multi-disciplinary approach in line with an enabling and rehabilitative focus.

The ultimate aim of the project was to reduce the incidence of falls by promoting decision making and staff autonomy, equipping staff with skills, knowledge and tools to promote patient safety, dignity choice and independence. A key part of this was increasing the confidence of staff in the identification of appropriate falls interventions, taking into account individual patient preferences.

In 2016 a multi-disciplinary falls working party was established. The working party have regularly audited falls and documentation, linked with community services, developed documentation and care plans, updated knowledge, training and policy, and collaborated with internal teams. Currently a falls documentation audit is taking place.

The joint development and introduction of documentation has been a success, addressing concerns raised by staff and providing them with the tools needed to practically promote choice and independence. Since the introduction of the falls working party there has been a considerable increase in communication regarding falls and collaborative working as well as a reduction in number of falls and level of harm obtained from falls over the last three years. A large proportion of falls have been identified as unavoidable as part of the audit work undertaken, but staff have been more pro-active in managing falls and maintaining levels of patient independence and are reporting increased levels of confidence in promoting patient choice whilst maintaining autonomy.

\section{P-127 ENHANCING PATIENT EXPERIENCE - FALLS PREVENTION WITHIN A HOSPICE}

Jennifer Dacombe, Kerri McManus. Countess Mountbatten House, Southampton, UK

10.1136/bmjspcare-2018-hospiceabs. 152

Patient safety is a key domain of quality care within the hospice setting. It is important to ensure clinical excellence and promoting safety through standards of practice. It is widely acknowledged and accepted within the palliative care setting that the progressive deterioration of both cognitive and physical/motor skills with disease process, treatment effects, and aging means falls are increasingly common. In addition patient autonomy becomes more important as they seek independence and quality of life as they deteriorate and death nears.

Standards Countess Mountbatten House is a 27-bedded inpatient unit. All patients on admission are assessed using a Falls Risk Assessment Tool due to their clinical condition and treatments all patients are highlighted as a high risk of falling and this is reflected in the recent data.

Data May 2017- April 2018 Countess Mountbatten House inpatient unit had 539 admissions with total of 86 patient falls, 59 of which were unwitnessed, 11 from patients walking/standing and 10 from falling from bed to floor (majority onto falls mats as part of the falls prevention plan of care). This a 25\% reduction in patients falls compared to May 2016 - April 2017 when 503 patients were admitted with a total of 115 patient falls recorded, 74 of which were unwitnessed, 18 from patients walking/standing and 12 falling from a bed to floor. This demonstrates evidence best practice, prevention and management.

Falls management How have we achieved this improvement in our patient care? Observation bay created introducing bay watch, increased training and education, empowering staff at all levels to recognise high falls risks, act upon changes in patient condition promptly, improved collaborative working with families, low threshold for additional staff to provide 1:1 care, improved communication and initiatives with multi- professional team. Use of sensor and motion mats and turnaround project.

\section{P-128 ALL ABOUT ME: THE PATIENT AS A PERSON IN PALLIATIVE CARE}

Emma Austen, Sarah Wheatland, Sue Timms, Michael Partridge. St Michael's Hospice, Basingstoke, UK

\subsection{6/bmjspcare-2018-hospiceabs. 153}

Background Patients with serious illness are at a risk of depersonalised, over-medicalised care as they move into later life (Shippee, Shippee, Mobley et al., 2018). Research shows the importance of the expression of feelings and being seen as an individual during the advanced stages of illness (Kennedy, 2016; Sampson, Finlay, Byrne et al., 2014).

Aim To evaluate our 'This is Me' white-boards from patient, relatives' and staff perspectives to identify their contribution to a person-centred approach in the hospice.

Methods Boards in patients' rooms were re-branded 'This is Me' boards. Patients and relatives were encouraged to write and draw on them, creating an expression of them as a person. Questionnaire feedback was analysed and themes identified (May-June 2018).

Results Patients and relatives:

- Patients felt it helped staff view them more as a person

- Provided a motivational function and a timetable

- Made the environment more personal and homely.

Staff:

- Informality and freedom of expression to project a new perspective

- The creativity deployed was uplifting

- Highlighted the uniqueness of each patient

- Power of visual imagery to inform care. 
Conclusion Evaluation of the 'This is Me' boards demonstrates a dynamic approach to patient-centred care where individual wishes and goals are communicated to all staff, and relatives in a meaningful patient-led approach.

\section{P-129 WHAT'S IMPORTANT TO ME}

Caroline Betts, Tracey Young, Lucy Kralj, Katherine Gogarty. St Raphael's Hospice, London, UK

\subsection{6/bmjspcare-2018-hospiceabs. 154}

Background Patients want to be viewed as an individual with dignity and respect. In the busy day-to-day of caring for people it was identified that staff were focusing on tasks of care rather than on what was meaningful for the people in their care.

The project began with a desire to involve patients in their care by identifying what is important to them. Utilising time in a different way to engage people in conversations giving them permission to tell us what is important to them; enabling the person to be truly involved in decisions about their care and informing their choices.

A team from the ward including a staff nurse as psychoanalyst, the practice development facilitator and Matron led the project. It became apparent that it was not going to be straightforward to integrate the project into everyday practice as it was identified that staff felt uncomfortable instigating conversations, referring to patients by room numbers or diagnosis. The culture on the ward had to be influenced and changed.

Aims To positively influence culture change on the ward. To focus the team's attention on the individual and what is important to them. To role model and support how conversations can be facilitated.

Methods Introduction of a new role led by the nurse psychoanalyst, to support team facilitation of meaningful conversations with patients. White boards installed in rooms to enable patients' families to write what is important to them. Handovers discuss patient communication and notations on the white boards to influence care.

Results The new nurse psychoanalyst role is positively impacting on the team practice. Improved documentation regarding patient family conversations demonstrates the culture of reticence to engage is changing. Patients' families are actively engaging in writing what is important to them on the white boards influencing their care.

\section{P-130 THE PALLIATIVE PATIENT'S ONGOING EXERCISE BEHAVIOURS POST HOSPICE GYM INPUT}

${ }^{1}$ Lara Cowley, ${ }^{2}$ Angela Glynn. 'St Wilfrid's Hospice, Eastbourne, UK; ${ }^{2}$ University of Brighton, Eastbourne, UK

\subsection{6/bmjspcare-2018-hospiceabs. 155}

Background With research demonstrating the benefit of exercise therapy in palliative care there is the need for exercise behaviours to continue ongoing in order to sustain the benefits long term. It is imperative hospices understand the palliative patient's self-managed exercise behaviours once they have completed gym programmes in order to best facilitate a positive uptake. Research exploring these behaviours in healthy populations and in those with a non-palliative diagnosis have found there are internal and external barriers to active uptake of exercise. These include patient understanding, education, motivation, health status, previous exercise behaviours and access to appropriate facilities or equipment.

Aim To explore the effects that participation in a hospice gym-based exercise programme has on ongoing exercise habits. Methods Qualitative study - focus group.

Results Findings demonstrated there was low uptake of ongoing exercise behaviours despite patients feeling the ability to stay independent gave meaning to their lives and identified exercise as a way of achieving this. Barriers included poor education on why ongoing exercise was important, poor motivation to do exercises at home, and practical issues such as access to transport and equipment. A pertinent finding was the patient's belief that the hospice staff were the only ones who were capable of managing their needs compared to other services. Coming to the hospice filled a void in their life and they formed a strong bond with their local centre.

Conclusions There were similar findings for the barriers to ongoing exercise behaviours in palliative patients and those with a non-terminal diagnosis. However, there are added complexities with palliative patients and the bond they develop with their hospice which can hinder active uptake of exercise in the community. More research is needed to clarify findings and explore facilitators of self-managed exercise in this patient group.

\section{P-131 HEALTHCARE PROFESSIONALS' VIEWS OF REHABILITATIVE PALLIATIVE CARE}

Olivia Beeney-Bennett. St. Wilfrid's Hospice, Eastbourne, UK

\subsection{6/bmjspcare-2018-hospiceabs.156}

Background As people in the UK are living longer with incurable diseases, it has been acknowledged that hospices need to review their provision of care (Calanzani, Higginson $\&$ Gomes, 2013). The adoption of a rehabilitative palliative care approach is recommended, which aims to optimise people's function, wellbeing and independence within the limitations of an advancing illness (Tiberini \& Richardson, 2015). There is limited research examining the perspective of healthcare professionals (HCPs) working in hospices regarding rehabilitative palliative care (Wosahlo \& Maddocks, 2015).

Aims To explore the views and experiences of HCPs working in hospice in-patient settings of rehabilitative palliative care to inform practice in hospice in-patient units.

Methods Jan-March 2017: Literature review and ethics application. April-June 2017: recruitment from two hospices. Qualitative approach: semi-structured interviews with HCPs working in hospice in-patient settings. Topic guide and field notes used. June-Sept 2017: interview audio-recordings transcribed verbatim, systematically analysed using Iterative Categorization (Neale, 2016) and themes developed from the data. Results 18 interviews conducted: nine nurses, three healthcare assistants, two doctors, two physiotherapists and two occupational therapists. Themes identified included a shared common understanding of rehabilitative palliative care and highlighted that aspects of this approach were practised within hospices. Barriers and enablers to rehabilitative palliative care were identified, including multidisciplinary team work and communication, the concept of 'tucking up' patients, the patient and family, and external perceptions of hospices. Healthcare 